\title{
Hip and knee arthroplasty waiting list - how accurate and fair?
}

\author{
T Kavalieratos, ${ }^{1}$ 2nd-year medical student; $\mathbf{M}$ Nortje, ${ }^{2}$ MB ChB, MMed (Orth), FCS (Orth); R N Dunn, ${ }^{3}$ MB ChB, MMed (Orth), \\ FCS (SA) Orth \\ ${ }^{1}$ Division of Orthopaedic Surgery, Faculty of Health Sciences, University of Cape Town, South Africa \\ ${ }^{2}$ Division of Orthopaedic Surgery, Faculty of Health Sciences, University of Cape Town and Groote Schuur Hospital, South Africa \\ ${ }^{3}$ HOD/Pieter Moll and Nuffield Chair, Division of Orthopaedic Surgery, Faculty of Health Sciences, University of Cape Town and \\ Groote Schuur Hospital, South Africa
}

Corresponding author: R N Dunn (robert.dunn@uct.ac.za)

\begin{abstract}
Background. Resource-intensive procedures require the use of patient waiting lists in an attempt to increase fairness of access to surgery and improve surgical efficiency. Total hip and knee arthroplasty has waiting lists in excess of years.

Objectives. To analyse our tertiary state institution's hip and knee arthroplasty waiting list to assess its accuracy.

Methods. At Groote Schuur Hospital, our hospital-maintained database was compared with the surgeons' personally maintained database. Patients were then telephoned to confirm their contactability, and to discover whether they still wanted the procedure, or if they had already had it. Waiting duration and patient demographics were then calculated.

Results. Of the 655 patients on the hospital waiting list, only 454 were contactable. Three hundred and nine patients still wanted the surgery, 93 had already undergone surgery and 52 no longer wanted surgery. The last group was the oldest and had had the longest waiting time. Those still waiting had waited 451 days (minimum - maximum (standard deviation), 90 - 1593 (228.5)), those that had had surgery 371 days (0 - 1728 (296)) and those no longer interested 523 days (138 - 1881 (260.9)). A total of 429 patients were present on the surgeons' list but not on the hospital list. They had had longer waiting times than those on the hospital list.

Conclusion. The arthroplasty waiting list is inaccurate due to the existence of two concurrent lists and poor data management, particularly of current contact details. The unfairness of a wide range of waiting times was identified, with patients only on the surgeons' personal database disadvantaged. These deficiencies have prompted the introduction of a scoring-based prioritisation system incorporating clinical, radiographic and societal parameters, in an effort to improve fair and appropriate access to this high-cost care.
\end{abstract}

S Afr Med J 2017;107(4):323-326. DOI:10.7196/SAMJ.2017.v107i4.12145

Orthopaedic surgery is an expensive procedure with high patient demand for reconstructive service. This mismatch between patient demand and available service results in the requirement for surgical waiting lists. Hip- and knee-joint replacement surgery (arthroplasty) is a life-changing procedure in terms of pain reduction and functional improvement, but patients can wait months to years for the procedure. This is not unique to South Africa (SA), but local challenges of high and competing trauma and infection, and burden of disease exacerbate the problem. ${ }^{[1]}$

These waiting lists are frequently used to determine demand and access by hospital managers and provincial planners. It is therefore necessary to understand the waiting list system and assess how accurately it reflects patient demand. At Groote Schuur Hospital (GSH), despite performing $>250$ hip- and knee-joint replacements per year, there is an extensive and growing waiting list.

Traditionally, waiting lists have been personally maintained by the surgeons involved using a variety of paper-based and electronic methods. Recently, our institution developed a hospitalbased electronic waiting list system for arthroplasty, requiring the interaction of administration and clinical staff. This has brought about many unforeseen challenges.

\section{Objectives}

We undertook to analyse our tertiary state institution's hip and knee arthroplasty waiting list to assess its accuracy.

\section{Methods}

At GSH, in the Department of Orthopaedic Surgery, surgeons have historically maintained personal waiting lists on an Excel (Microsoft, USA) spreadsheet. The hospital recently introduced a Clinicombased (CompuGroup, Slovakia) electronic waiting list, where clerks are responsible for maintaining the data. However, owing to the surgeons' circumspection, both lists were still being maintained during the consolidation period.

The hospital-maintained database was interrogated at the end of November 2015, and an Excel spreadsheet was generated with all patients on the waiting list at the time. This dataset included their name, hospital number, contact details, date of birth, date when placed on the waiting list and whether it was for a hip or knee replacement.

We then compared the hospital list with the list personally maintained by the arthroplasty surgeons, to identify any patients who were missing from the hospital list.

All the patients on the hospital list were contacted telephonically. Three repeated attempts were made on different days at different times before a patient was classed as 'not contactable'. If the patient was not contactable, the hospital system was interrogated to see whether the patient was still active in the outpatient system between December 2015 and June 2016. Once contacted, the patient was asked if they still wanted the booked procedure, and if not, why not. 
Patients on the surgeons' personally maintained list and not appearing on the hospital list were tracked on the state hospital Clinicom system regarding theatre activity.

The data were analysed for the duration patients had waited so far. This was calculated by determining the number of days they had waited from when placed on the waiting list to the last day we called patients (30 November 2015). Age demographics were compared for the groups who wanted to remain on the list as opposed to those no longer wanting the surgery, as well as those who had in fact undergone the procedure but had not been taken off the list. These differences were assessed statistically with an unpaired $t$-test.

\section{Results}

There were 655 patients on the hospital list (Fig. 1). An additional 429 cases were identified on the surgeons' personal list that were absent from the hospital list; 162 names were present on the hospital list that were absent from the surgeons' personal list.

Of the 655 patients on the hospital list, 454 (69\%) were contactable and 201 (31\%) were non-contactable. Of the 201 patients who were not contactable, we confirmed via the outpatient booking system that 88 had attended the clinic in the last 7 months. Therefore, 542 patients $(83 \%)$ were still visibly active on the state waiting list.

Of the 454 contactable patients, 309 patients (68\%) still wanted to have the surgery. Fifty-two patients (11\%) were no longer interested in having the surgery and requested to be removed from the waiting list. Ninety-three patients $(20 \%)$ had already had the surgery performed.

The main reasons for the 52 patients no longer wanting surgery were that they no longer had pain or were no longer interested in the surgery (Table 1).

The mean age of the patients who wanted to be taken off the list was 67 years $(37-84(10.5))$, the patients who still wanted to be on the list averaged 62 years $(23-83(11.0))$, and the patients who had already had the surgery but were not removed from the list averaged 64 years $(30-91(12.0))$. The patients who wanted to be taken off the list were on average significantly older than the group that still wanted surgery $(p=0.0009)$.

Of the 93 patients who had already undergone surgery but remained on the list, 71 had had their surgery at GSH, while 22 had decided to have their surgery elsewhere, primarily at private hospitals.

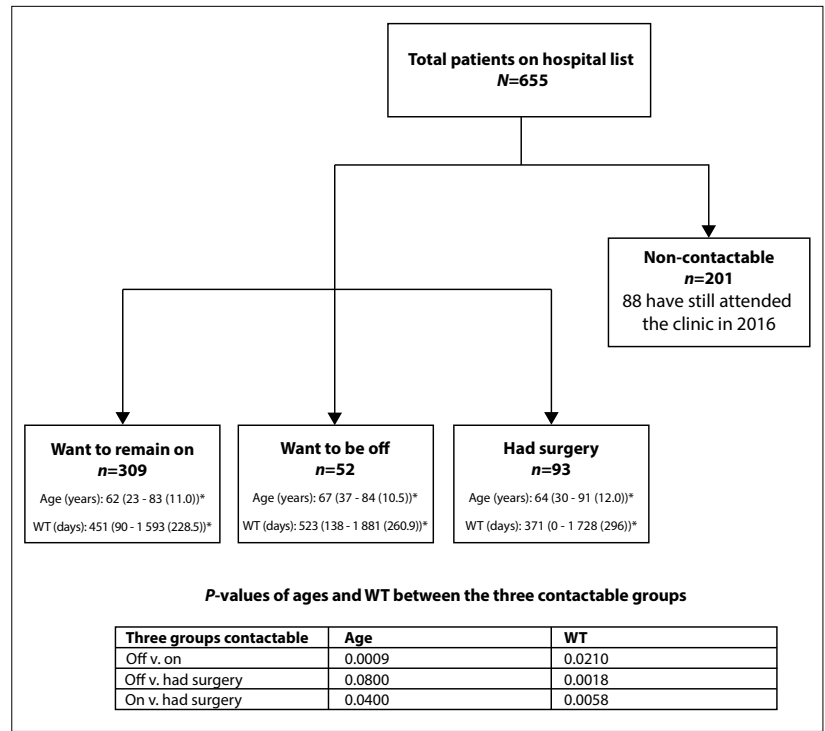

Fig. 1. Findings from the hospital list with average age and WT for each group. $\left(W T=\right.$ waiting time. ${ }^{*}$ mean (range $(S D)$.)
The time on the waiting list was calculated from the time up to 30 November 2015, the date of our data collection. For those who had in fact had their surgery but erroneously remained on the list, the waiting time was calculated from the time they were placed on the list to their individual date of surgery. This latter group had had a significantly shorter wait than those still waiting to have their surgery $(p=0.0058)$. They waited 371 days $(0-1728$ (296)). Patients still waiting to have their surgery waited 451 days (90 - 1593 (228.5)). The patients who indicated that they no longer wanted surgery had waited $\sim 523$ days (138 - 1881 (260.9)).

When reviewing the 429 patients who were on the surgeons' personal list, but not present on the state list, 98 patients had already had surgery, having waited an average of 990 days. The patients still waiting for their surgery had waited on average 1662 days.

There was a significant difference between the patients who had already had the surgery in the hospital and were on the surgeons' list $(p<0001)$, and the patients who were still waiting for the surgery $(p<0001)$.

When comparing hip and knee arthroplasty, knee patients had waited on average 461 (90 - 1513 (222.8)) days, while patients waiting for hip replacements waited on average 412 (104 - 1593 (242.3)) days $(p=0.06)$.

\section{Discussion}

Waiting lists are necessary to improve fair access to healthcare such as high-cost surgical procedures. They should ensure the optimal use of time for the healthcare workers and patients. Ideally, patients should be stratified by means of a predetermined scoring system to match needs, skills and outcomes. However, many waiting lists are poorly managed, and anecdotal experience suggests that fairness to access is impeded by difficulty contacting patients, owing to inaccurate contact details and the surgeons' practice of calling in the last seen patient when there is a last-minute cancellation. However, clinicians and services are increasingly being assessed by their waiting periods and lists, especially by increasing numbers of hospital management staff.

Patients waiting longer than 6 months for their surgery have been shown to experience greater preoperative anxiety, depression and dissatisfaction than those waiting less than 6 months. ${ }^{[2]}$ Generally, neither pain nor function seemed to worsen for patients waiting less than 6 months for knee or hip replacements. ${ }^{[3]}$

Our hospital waiting list had a wide range of waiting times, which ranged from 0 to 1880 days. The mean waiting time for all groups was longer than 6 months. Although we did not investigate the patients'

Table 1. Reasons given by patients who wanted to be taken off the hospital waiting list

\begin{tabular}{ll}
\hline Reason for wanting to be taken off list & Respondents, $\boldsymbol{n}$ \\
\hline No longer felt pain & 19 \\
Not interested any more & 8 \\
Died & 4 \\
Too old & 4 \\
Too sick & 4 \\
Operation cancelled & 3 \\
Scared & 3 \\
Felt they had waited too long & 2 \\
No reason given & 5 \\
Total & 52
\end{tabular}


psychological and emotional status, this may have contributed to the $11 \%$ who requested to be removed from the waiting list.

Conner-Spady et al. ${ }^{[4]}$ found that patients thought that the maximum acceptable waiting time should be 4 months, well below the actual times found in this study. Conner-Spady et al..$^{[4}$ reported an ideal waiting time of 2 months, where patients could prepare for the surgery emotionally and physically and get their affairs in order.

In our study, the $20 \%$ of patients who had already had the surgery but were not taken off the list had had the shortest mean waiting time. The majority of these patients had had their operation at GSH, while the rest had resorted to private hospitals. Our unit offers privately funded additional lists when the funding is made available by corporate and philanthropic partners. Suitable, low-risk patients on these additional lists are treated at GSH and are taken off the standard waiting list. It appears that the administration did not keep up and remove the patients from the list as they did not follow the usual admission procedure. Other patients were treated at our satellite hospitals, indicating that some patients consult at different entry points to the system. A few took advantage of lowcost philanthropic options provided by our sessional staff through a not-for-profit trust.

The patients no longer wanting surgery and opting to be taken off the list were significantly older than the rest. In fact, their reasons for this decision were often that they felt too old or too sickly. Surgeons need to effectively council patients regarding surgery before adding them to the waiting list, rather than be preoccupied with radiographical changes, to avoid this situation.

Many of the patients on the state waiting list were non-contactable. This was due to the wrong telephone numbers being recorded, outdated contact information and in some cases no contact information at all. This highlights the need for active data management and regular updating of information when patients present at the various clinics. This has improved somewhat with mobile phones and the Regulation of Interception of Communications and Provision of Communication-Related Information Act (RICA) requirements, which result in less frequent change in mobile numbers.

Owing to surgeons' reluctance to trust the newer hospital-based electronic waiting list, they have continued to use a concurrent personally maintained list. However, poor co-ordination has resulted in neither list being representative of all the patients waiting. It appears that patients on the surgeons' personal list and not on the hospital list were disadvantaged by longer waiting times. This may be due to reduced access by all parties to the personal list.

In order for the waiting-list system to work optimally, surgeons need to adopt a single hospital-based waiting list that is actively managed by competent administration staff. It has been shown that change and maintenance are best improved when the surgeons 'buy into' the system, are involved in strategy planning and have a sense of shared leadership. ${ }^{[5]}$

Ackerman et al. ${ }^{[3]}$ reported that the mean waiting time for knee replacements was longer than that for hip replacements. Although showing only a mean difference of 6 weeks, our study echoes this. This may well be due to the slightly longer duration of knee replacement surgery. As many theatre allocations are based on a morning or afternoon list, it prevents two knees being done on one list, whereas replacing two hips is possible. Clearly, in order to reduce the waiting-list time, more resources are needed in the orthopaedic department to increase the throughput.

Waiting lists improve equality and fairness of access to surgery by using a prioritisation system based on multiple demographic and clinical factors. Prioritisation is important because it allows patients to wait a shorter amount of time on the list according to their need for the surgery, which improves fairness. ${ }^{[6]}$ One study found that $84 \%$ of waiting patients felt that it was fair if another patient was placed ahead of them if they had worse pain or greater limitation of daily functions. ${ }^{[4]}$

However, Tebé et al. ${ }^{[7]}$ reported on primary knee arthroplasty waiting lists and concluded that the priority system had no effect on implementing prioritisation for patients based on their severity.

Our study showed a broad range of patients' waiting times, from a few days to several years. Although this may be based on ad hoc clinical prioritisation, it is more likely owing to patient contactability and short notice availability, which is unfair in terms of access to care. This may be due to the surgeons having a tendency to call the last patient they remember consulting with when an unexpected slot opens, instead of phoning the next patient on the waiting list. Despite this, we can also hypothesise that the more extreme waiting times are outliers, which may mean that some of the patients who have been waiting for years have stopped coming to the clinic but were never removed from the waiting list.

The problem with the state waiting list is going to take time and resources to amend, even if there is immediate action in improving our administration system. Therefore, it is important to think of strategies that may be advantageous to patients who are waiting long times for their surgeries. Some studies have found that there is a deterioration in patients who have to wait a long time for their surgeries, in terms of functional limitations and pain. ${ }^{[8]}$ In an attempt to combat this problem, several Australian states have implemented a system that addresses the issues of long waiting times and prioritisation. ${ }^{[9]}$ They have appointed musculoskeletal co-ordinators to routinely assess patients who may be experiencing deterioration in function prior to their surgery, and to determine whether they need to be placed higher on the list or need emergency intervention. This is a productive strategy as it strengthens the prioritisation system and reduces waiting times for those patients who are most in need.

Another practice that may reduce patient default is the implementation of a maximum waiting time for patients, dependent on their priority score. ${ }^{[7]}$ An open-ended amount of time may leave the patient feeling hopeless. ${ }^{[4]}$ Therefore, if some idea of how long they will have to wait is provided, the probability of the patient remaining on the list will be increased. The deficiencies identified in this study prompted our unit to introduce a scoring-based prioritisation system incorporating clinical, radiographic and societal parameters in an effort to improve fair and appropriate access to high-cost care.

\section{Conclusion}

This study confirms that the current arthroplasty waiting list is inaccurate, owing to the existence of two concurrent lists, and to poor data management in terms of current patient contact details. It highlights the long waiting times, with the wide range in such times experienced by patients. Those patients who were only on the surgeons' personal database were disadvantaged by even longer waits.

A single, actively managed, electronic system integrated into the hospital's system for accurate demographic data is recommended. However, this needs surgeon confidence and buy-in. With this, further predetermined clinical, radiographic and societal parameters can be utilised to prioritise the patients and improve fair and appropriate access to high-cost care. 
Acknowledgement. The authors would like to thank Ms Noluthando Marotola for her administrative help in tirelessly calling all the patients on the waiting list.

1. Dunn RN. Musculoskeletal burden of disease: Do we have any idea? SA Orthopaedic J 2012;11(2):20-22.

2. Lizaur-Utrilla A, Martinez-Mendez D, Miralles-Muñoz F, Marco-Gomez L, Lopez-Prats F. Negative impact of waiting time for primary total knee arthroplasty on satisfaction and patient-reported outcome. Int Orthop 2016;40(11):2303-2307. https://doi.org/10.1007/s00264-016-3209-0

3. Ackerman I, Bennell K, Osborne R. Decline in health-related quality of life reported by more than half of those waiting for joint replacement surgery: A prospective cohort study. BMC Musculoskelet Disord 2011;12(1):2-7. https://doi.org/10.1186/1471-2474-12-108

4. Conner-Spady B, Sanmartin C, Johnston G, McGurran J, Kehler M, Noseworthy T. 'There are too many of us to fix'. Patients' views of acceptable waiting times for hip and knee replacement. J Health Sery Res Policy 2009;14(4):212-218. https://doi.org/10.1258/jhsrp.2009.00812

5. Amar C, Pomey M, SanMartin C, De Coster C, Noseworthy T. Sustainability: Orthopaedic surgery wait time management strategies. Int J Health Care Qual Assur 2015;28(4):1-11. https://doi.org/10.1108/ IJHCQA-11-2013-0131
6. Román R, Comas M, Mar J, Bernal E, et al. Geographical variations in the benefit of applying a prioritization system for cataract surgery in different regions of Spain. BMC Health Serv Res 2008;8(1):8-32. https://doi.org/10.1186/1472-6963-8-32

7. Tebé C, Comas M, Adam P, Solans-Domènech M, Allepuz A, Espallargues M. Impact of a priority system on patients in waiting lists for knee arthroplasty. J Eval Clin Pract 2014;21(1):91-96. https://
s. doi.org/10.1111/jep. 12248

8. Desmeules F, Dionne C, Belzile É, Bourbonnais R, Frémont P. The impacts of pre-surgery wait for total knee replacement on pain, function and health-related quality of life six months after surgery. J Eval Clin Pract 2010;18(1):111-120. https://doi.org/10.1111/j.1365-2753.2010.01541.x

9. Witt J, Scott A, Osborne R. Designing choice experiments with many attributes. An application to setting priorities for orthopaedic waiting lists. Health Econ 2009;8(6):681-696. https://doi. org $/ 10.1002 /$ hec. 1396

Accepted 16 January 2017 